Pacific

Journal of

Mathematics

POSITIVITY RESULTS FOR THE YANG-MILLS-HIGGS HESSIAN

George Androulakis and Stamatis Dostoglou

Volume 194 No. 1

May 2000 


\title{
POSITIVITY RESULTS FOR THE YANG-MILLS-HIGGS HESSIAN
}

\author{
George Androulakis and Stamatis Dostoglou
}

\begin{abstract}
We study the second derivative $Q_{c_{\lambda}}$ of the Yang-MillsHiggs functional with structure group $S U(2)$ at a spherically symmetric critical point $c_{\lambda}$ when the self-interaction parameter $\lambda$ is not close to 0 . We find that if $Q_{c_{\lambda_{0}}}$ is non-negative and with kernel consisting entirely of translation modes then positivity persists in a neighborhood of $\lambda_{0}$. In particular, we show that if translation modes always account for the whole kernel then $Q_{c_{\lambda}}$ is always non-negative. This extends our previous results for $\lambda$ in an neighborhood of 0 .
\end{abstract}

\section{Introduction.}

The Yang-Mills-Higgs functional $E_{\lambda}$ on $\mathbb{R}^{3}$, with structure group $S U(2)$, is the classical static version of the functional introduced by P. Higgs in $[\mathbf{H}]$. The critical points of $E_{\lambda}$ correspond to magnetic monopoles.

It has been known since the late 70 s that spherically symmetric critical points $c_{\lambda}$ of $E_{\lambda}$ exist for all values of the positive parameter $\lambda$. The authors of this paper have recently shown that for positive $\lambda$ in a neighborhood of 0 these critical points have non-negative Hessian $Q_{c_{\lambda}}$, i.e., they are (weakly) stable, see $[\mathbf{A D}]$. The aim of this article is to investigate the stability of $c_{\lambda}$ for (the more relevant for physics) large values of $\lambda$.

For $\lambda=0$ the spherically symmetric solution $c_{0}$ satisfies the first order (Bogomol'nyi) equation for global minima, $[\mathbf{J T}]$. This equation is unique to the $\lambda=0$ case and has no analogue for $\lambda \neq 0$. One of the crucial observations in $[\mathbf{A D}]$ for extending the positivity from $\lambda=0$ to $\lambda \neq 0$ is that the kernel of the Hessian at 0 consists entirely of translation modes. Here we show that this is not special to $\lambda=0$ : For any positive $\lambda_{0}$ where $Q_{c_{\lambda_{0}}}$ is non-negative, if the kernel of $Q_{c_{\lambda_{0}}}$ is the span of the translation modes then $Q_{c_{\lambda}}$ is non-negative for $\lambda$ in a neighborhood of $\lambda_{0}$. In the same neighborhood the dimension of the kernel may not increase. The first main result then is:

Theorem. The set of $\lambda \geq 0$ for which $Q_{c_{\lambda}}$ is non-negative and has 3dimensional kernel is open. 
In addition, since for $\lambda$ close to $\lambda_{0}$ it is shown that $Q_{c_{\lambda}}$ is close to $Q_{c_{\lambda_{0}}}$, the set of $\lambda$ 's for which $Q_{c_{\lambda}} \geq 0$ is closed, yielding:

Corollary. If $\operatorname{ker} Q_{c_{\lambda}}$ is always 3-dimensional then $Q_{c_{\lambda}}$ is non-negative for all $\lambda$.

The proof of the main results consists of three parts. First it is shown that if $Q_{c_{\lambda_{0}}}$ is non-negative then, away from its kernel, it is bounded below by a strictly positive constant. Then $c_{\lambda}$ is shown to converge to $c_{\lambda_{0}}$ in the configuration space as $\lambda \rightarrow \lambda_{0}$. This implies that the Hessians $Q_{c_{\lambda}}$ are all defined on the same Hilbert space and that they differ by a small amount. It is then shown that the subspaces $N_{\lambda}$ spanned by the translation modes of $c_{\lambda}$ contain the kernel of $Q_{c_{\lambda_{0}}}$ at the limit. This implies that directions orthogonal to $N_{\lambda}$ are almost orthogonal to, and definitely not in, the kernel of $Q_{c_{\lambda_{0}}}$. For the last two steps in the proof the estimates of [AD] need to be extended from uniform estimates on some neighborhood of 0 to uniform estimates on any compact $\lambda$-interval.

In Section 2 we review the basics of the theory and state the main results. The proofs are contained in Section 3, where we focus on aspects that are genuinely different from the $\lambda_{0}=0$ case. It is in Section 4 that we show how to improve the estimates in $[\mathbf{A D}]$ so that they hold on any bounded $\lambda$-interval.

\section{The functional $E_{\lambda}$ and the symmetric solutions $c_{\lambda}$.}

The Yang-Mills-Higgs functional $E_{\lambda}$ with self-interaction parameter $\lambda \geq 0$ is defined by

$$
E_{\lambda}(A, \Phi)=\frac{1}{2} \int_{\mathbf{R}^{3}}\left\{\left|F_{A}\right|^{2}+\left|d_{A} \Phi\right|^{2}+\frac{\lambda}{4}\left(|\Phi|^{2}-1\right)^{2}\right\} d^{3} x,
$$

on pairs $c=(A, \Phi)$. Here $A$ is a connection on the $S U(2)$ bundle $S U(2) \times \mathbf{R}^{3}$ over $\mathbf{R}^{3}$ and $\Phi$ is a section of the associated bundle $E$ with fiber the Lie Algebra $\mathfrak{s} u(2), E=\mathfrak{s} u(2) \times \mathbf{R}^{3} . F_{A}$ is the curvature of the connection $A$ and $d_{A} \Phi$ the covariant derivative of $\Phi$ with respect to the connection $A$ :

$$
F_{A}=d A+\frac{1}{2}[A, A], \quad d_{A} \Phi=d \Phi+[A, \Phi] .
$$

All norms use the Killing inner product on $\mathfrak{s} u(2)$ and the standard metric on $\mathbf{R}^{3}$.

$E_{\lambda}$ is defined on the configuration space

$$
\hat{\mathcal{C}}=\left\{(A, \Phi): A \in L_{1, \mathrm{loc}}^{2}, \Phi \in L_{1, \mathrm{loc}}^{2}, E_{\lambda}(A, \Phi)<\infty .\right\}
$$

Note here that $\hat{\mathcal{C}}$ stays the same for all $\lambda>0$. For the special case $\lambda=0$ see $[\mathrm{AD}] . \hat{\mathcal{C}}$ is equipped with the $L_{1, \text { loc }}^{2}$ topology intersected with the topology 
that makes $\left\|d_{A} \Phi\right\|_{2}$ and $\left\|F_{A}\right\|_{2}$ continuous. $E_{\lambda}, \lambda \geq 0$ is invariant under the action of the gauge group

$$
\mathcal{G}=\left\{g: \mathbf{R}^{3} \rightarrow \mathrm{SU}(2), \quad \mathrm{g} \in \mathrm{L}_{2, \mathrm{loc}}^{2}\right\},
$$

where $g \cdot A=g A g^{-1}+g d g^{-1}$, and $g \cdot \Phi=g \Phi g^{-1}$. Then $E_{\lambda}$ descends to the quotient $\mathcal{C}=\hat{\mathcal{C}} / \mathcal{G}$.

To define the space $T_{c} \mathcal{C}$ of admissible infinitesimal perturbations at $c=$ $(A, \Phi)$ in $\mathcal{C}$, consider first the completion $H_{c}$ of $C_{0}^{\infty}$ sections on $\mathbb{R}^{3}$ with respect to the inner product norm

$$
\|(a, \phi)\|_{c}^{2}=\left\|\nabla_{A} a\right\|_{2}^{2}+\left\|\nabla_{A} \phi\right\|_{2}^{2}+\|[\Phi, a]\|_{2}^{2}+\|\phi\|_{2}^{2} .
$$

$H_{c}$ contains only directions that keep $E_{\lambda}$ finite, c.f. [T1], but it still contains deformations along the orbit of $\mathcal{G}$. This is remedied here by excluding the elements of the kernel of the (formal) adjoint of the linearization of the action

$$
\partial_{c}(a, \phi)=-d_{A}^{*} a+[\Phi, \phi]
$$

We define therefore

$$
T_{c} \mathcal{C}=\left\{(a, \phi) \in H_{c}: \partial_{c}(a, \phi)=0\right\} .
$$

The variational equations for $\lambda \geq 0$ are the Yang-Mills-Higgs equations

$$
d_{A}^{*} F_{A}=\left[d_{A} \Phi, \Phi\right], \quad d_{A}^{*} d_{A} \Phi=-\frac{\lambda}{2} \Phi\left(|\Phi|^{2}-1\right) .
$$

For any $c=(A, \Phi)$ in $\mathcal{C}$, the second derivative of the energy $E_{\lambda}$ defines a bilinear form on $T_{c} \mathcal{C}$

$$
\begin{aligned}
& \hat{Q}_{\lambda, c}\left(\left(a_{1}, \phi_{1}\right),\left(a_{2}, \phi_{2}\right)\right) \\
& =\left.\frac{d^{2}}{d s d t}\right|_{(0,0)} E_{\lambda}\left(A+s a_{1}+t a_{2}, \Phi+s \phi_{1}+t \phi_{2}\right), \quad \lambda \geq 0,
\end{aligned}
$$

the Hessian of the Yang-Mills-Higgs functional. Then

$$
\begin{aligned}
\hat{Q}_{\lambda, c}\left(\left(a_{1}, \phi_{1}\right),\left(a_{2}, \phi_{2}\right)\right) & \\
= & \left\langle F_{A},\left[a_{1}, a_{2}\right]\right\rangle+\left\langle d_{A} \Phi,\left[a_{1}, \phi_{2}\right]+\left[a_{2}, \phi_{1}\right]\right\rangle+\left\langle d_{A} a_{1}, d_{A} a_{2}\right\rangle \\
& +\left\langle d_{A} \phi_{1}, d_{A} \phi_{2}\right\rangle+\left\langle\left[a_{1}, \Phi\right],\left[a_{2}, \Phi\right]\right\rangle+\left\langle d_{A} \phi_{1},\left[a_{2}, \Phi\right]\right\rangle \\
& +\left\langle d_{A} \phi_{2},\left[a_{1}, \Phi\right]\right\rangle+\frac{\lambda}{2} \int_{\mathbf{R}^{3}}\left(|\Phi|^{2}-1\right)\left\langle\phi_{1}, \phi_{2}\right\rangle d^{3} x \\
& +\lambda \int_{\mathbf{R}^{3}}\left\langle\Phi, \phi_{1}\right\rangle\left\langle\Phi, \phi_{2}\right\rangle d^{3} x
\end{aligned}
$$

and the corresponding quadratic form is

$$
\begin{aligned}
Q_{\lambda, c}(a, \phi)= & \left\|d_{A} a\right\|_{2}^{2}+\left\|d_{A} \phi\right\|_{2}^{2}+\|[a, \Phi]\|_{2}^{2} \\
& +\left\langle F_{A},[a, a]\right\rangle+2\left\langle d_{A} \Phi,[a, \phi]\right\rangle+2\left\langle d_{A} \phi,[a, \Phi]\right\rangle
\end{aligned}
$$




$$
+\frac{\lambda}{2} \int_{\mathbf{R}^{3}}\left(|\Phi|^{2}-1\right)|\phi|^{2} d^{3} x+\lambda \int_{\mathbf{R}^{3}}\langle\Phi, \phi\rangle^{2} d^{3} x .
$$

Now recall the following standard:

Definition 2.1. Let $\hat{Q}$ be a bilinear form a Hilbert space $H$. Then $v_{0}$ in $H$ is in $\operatorname{ker} \hat{Q}$ if and only if

$$
\hat{Q}\left(v_{0}, v\right)=0
$$

for all $v$ in $H$.

Throughout this paper whenever $Q$ is the quadratic form associated to a bilinear form $\hat{Q}$ we use the phrase " $v_{0}$ is in $\operatorname{ker} Q$ " to mean " $v_{0}$ is in $\operatorname{ker} \hat{Q}$ ".

The following is rewriting $Q_{\lambda, c}$ on $T_{c} \mathcal{C}$ as in [T2], page 246.

Lemma 2.2. For $(a, \phi)$ in $T_{c} \mathcal{C}$,

$$
\begin{aligned}
Q_{\lambda, c}(a, \phi)= & \left\|\nabla_{A} a\right\|_{2}^{2}+\left\|\nabla_{A} \phi\right\|_{2}^{2}+\|[a, \Phi]\|_{2}^{2}+\|[\Phi, \phi]\|_{2}^{2} \\
& +2\left\langle F_{A},[a, a]\right\rangle+4\left\langle d_{A} \Phi,[a, \phi]\right\rangle \\
& +\frac{\lambda}{2} \int_{\mathbf{R}^{3}}\left(|\Phi|^{2}-1\right)|\phi|^{2} d^{3} x+\lambda \int_{\mathbf{R}^{3}}\langle\Phi, \phi\rangle^{2} d^{3} x .
\end{aligned}
$$

Proof. First separate in $Q_{\lambda, c}$ the terms that contain $\lambda$ from those that do not, using the obvious notation:

$$
Q_{\lambda, c}(a, \phi)=Q_{0, c}(a, \phi)+\frac{\lambda}{2} \int_{\mathbf{R}^{3}}\left(|\Phi|^{2}-1\right)|\phi|^{2} d^{3} x+\lambda \int_{\mathbf{R}^{3}}\langle\Phi, \phi\rangle^{2} d^{3} x .
$$

Next use the Bochner-Weitzenböck formula for 1-forms on flat spaces, see page 95 of $[\mathbf{L}]$, and integrate by parts to get

$$
\left\|d_{A} a\right\|_{2}^{2}+\left\|d_{A}^{*} a\right\|_{2}^{2}=\left\|\nabla_{A} a\right\|_{2}^{2}+\left\langle F_{A},[a, a]\right\rangle .
$$

Then

$$
\begin{aligned}
Q_{0, c}(a, \phi)= & \left\|\nabla_{A} a\right\|_{2}^{2}-\left\|d_{A}^{*} a\right\|_{2}^{2}+\left\|d_{A} \phi\right\|_{2}^{2}+\|[a, \Phi]\|_{2}^{2} \\
& +2\left\langle F_{A},[a, a]\right\rangle+2\left\langle d_{A} \Phi,[a, \phi]\right\rangle+2\left\langle d_{A} \phi,[a, \Phi]\right\rangle .
\end{aligned}
$$

Therefore for $(a, \phi)$ in $T_{c} \mathcal{C}$, where $d_{A}^{*} a-[\Phi, \phi]=0$ holds,

$$
\begin{aligned}
Q_{0, c}(a, \phi)= & \left\|\nabla_{A} a\right\|_{2}^{2}+\left\|d_{A} \phi\right\|_{2}^{2}+\|[a, \Phi]\|_{2}^{2}+\|[\Phi, \phi]\|_{2}^{2} \\
& -2\|[\Phi, \phi]\|_{2}^{2}+2\left\langle F_{A},[a, a]\right\rangle+2\left\langle d_{A} \Phi,[a, \phi]\right\rangle+2\left\langle d_{A} \phi,[a, \Phi]\right\rangle .
\end{aligned}
$$

Now observe that on $T_{c} \mathcal{C}$ we also have

$$
\begin{aligned}
& \left\langle d_{A} \Phi,[a, \phi]\right\rangle \\
& =\sum_{1}^{3} \int_{\mathbb{R}^{3}}\left\langle\left(d_{A}\right)_{i} \Phi,\left[a_{i}, \phi\right]\right\rangle \\
& =-\sum_{1}^{3} \int_{\mathbb{R}^{3}}\left\langle\Phi,\left(d_{A}\right)_{i}\left[a_{i}, \phi\right]\right\rangle
\end{aligned}
$$




$$
\begin{aligned}
& =\int_{\mathbb{R}^{3}}\left\langle\Phi,\left[-\sum_{1}^{3}\left(d_{A}\right)_{i} a_{i}, \phi\right]\right\rangle-\sum_{1}^{3} \int_{\mathbb{R}^{3}}\left\langle\Phi,\left[a_{i},\left(d_{A}\right)_{i} \phi\right]\right\rangle \\
& =\int_{\mathbb{R}^{3}}\left\langle\Phi,\left[d_{A}^{*} a, \phi\right]\right\rangle-\int_{\mathbb{R}^{3}}\left\langle\Phi,\left[a, d_{A} \phi\right]\right\rangle \\
& =\int_{\mathbb{R}^{3}}\langle\Phi,[[\Phi, \phi], \phi]\rangle+\int_{\mathbb{R}^{3}}\left\langle[a, \Phi], d_{A} \phi\right\rangle \\
& =-\|[\Phi, \phi]\|_{2}^{2}+\left\langle d_{A} \phi,[a, \Phi]\right\rangle,
\end{aligned}
$$

to finally get

$$
\begin{aligned}
& Q_{0, c}(a, \phi) \\
& =\left\|\nabla_{A} a\right\|_{2}^{2}+\left\|d_{A} \phi\right\|_{2}^{2}+\|[a, \Phi]\|_{2}^{2}+\|[\Phi, \phi]\|_{2}^{2}+2\left\langle F_{A},[a, a]\right\rangle+4\left\langle d_{A} \Phi,[a, \phi]\right\rangle .
\end{aligned}
$$

In $[\mathbf{t H}]$ and $[\mathbf{P}]$ 't Hooft and Polyakov suggest spherically symmetric solutions for the three-dimensional Yang-Mills-Higgs equations. With respect to the standard basis $e_{a}, a=1,2,3$ of $\mathfrak{s} u(2)$ their Ansatz is

$$
A=\varepsilon_{i j a} \frac{x_{j}}{r^{2}}(1-K(r)) e_{a} d x_{i}, \quad \Phi=\frac{x_{\alpha}}{r} \frac{H(r)}{r} e_{a},
$$

with boundary conditions $K(r) \rightarrow 0$ and $H / r \rightarrow 1$, as $r \rightarrow \infty$.

On configurations of this form, $E_{\lambda}$ is

$$
\begin{aligned}
E_{\lambda}(H, K)=4 \pi \int_{0}^{\infty}\left\{\left(K^{\prime}\right)^{2}\right. & +\frac{1}{2}\left(H^{\prime}-\frac{H}{r}\right)^{2}+\frac{K^{2} H^{2}}{r^{2}}+\frac{1}{2} \frac{\left(K^{2}-1\right)^{2}}{r^{2}} \\
& \left.+\frac{\lambda}{4}\left(\frac{H^{2}}{r}-r\right)^{2}\right\} d r .
\end{aligned}
$$

A critical point $\left(K_{\lambda}, H_{\lambda}\right)$ of the 1-dimensional integral satisfies the variational equation

$$
\left.\frac{d}{d t}\right|_{t=0} E_{\lambda}\left(H_{\lambda}+t h, K_{\lambda}+t k\right)=0
$$

for all $h, k$ with compact support on $[0, \infty)$. This yields the system of non-linear, second order, ordinary differential equations

$$
\begin{aligned}
& K_{\lambda}^{\prime \prime}=\frac{H_{\lambda}^{2}-1+K_{\lambda}^{2}}{r^{2}} K_{\lambda} \\
& H_{\lambda}^{\prime \prime}=\frac{2 K_{\lambda}^{2}}{r^{2}} H_{\lambda}-4 \lambda H_{\lambda}\left(1-\frac{H_{\lambda}^{2}}{r^{2}}\right) .
\end{aligned}
$$

It is relatively easy to produce critical points of the 1-dimensional integral by direct minimization, see $[\mathbf{D}]$ for example. On the other hand it is a standard fact, referred to as "the principle of symmetric criticality" in $[\mathbf{P a}]$, 
that due to symmetry a critical point of the 1-dimensional integral is also a critical point of $E_{\lambda}$ overall.

Therefore for each $\lambda$ there is a spherically symmetric monopole solution. Throughout this paper

$$
c_{\lambda}=\left(K_{\lambda}, H_{\lambda}\right)
$$

will denote this solution.

Remark on notation. The notation for the Hessian $Q_{\lambda, c_{\lambda}}$ of $E_{\lambda}$ over all directions in $T_{c_{\lambda}} \mathcal{C}$ will be shortened to $Q_{c_{\lambda}}$. Otherwise, $Q_{\lambda, c}$ will denote the Hessian at an arbitrary configuration $c$ in $\mathcal{C}$.

There is no a priori reason why $c_{\lambda}$ should be an overall minimum. For example, the spherically symmetric minimizer of the Skyrmion functional has (non-spherically symmetric) unstable directions, see [WB].

For $\lambda=0$ the the point $c_{0}$ is a global minimum in the connected component of all configurations with finite $E_{0}$ energy, [M].

For $\lambda \neq 0$ and small, the main result in $[\mathbf{A D}]$ is:

Theorem 2.3. There is $\lambda_{0}>0$ such that $Q_{c_{\lambda}}(v) \geq 0$ for all $\lambda \leq \lambda_{0}$ and for all $v$ in $T_{c_{\lambda}} \mathcal{C}$. Furthermore,

$$
\operatorname{ker} Q_{c_{\lambda}}=\left\langle\frac{\partial c_{\lambda}}{\partial x_{i}}, i=1,2,3\right\rangle
$$

The behavior of $Q_{c_{\lambda}}$ for $\lambda$ away from $\lambda=0$ is investigated here. For this, define

$$
\begin{gathered}
\Lambda=\left\{\lambda>0: Q_{c_{\lambda}} \geq 0\right\} \\
\Lambda^{\prime}=\left\{\lambda \geq 0: \operatorname{ker} Q_{c_{\lambda}}=\left\langle\frac{\partial c_{\lambda}}{\partial x_{i}},\right\rangle_{i=1,2,3}\right\} .
\end{gathered}
$$

Then Theorem 2.3 states that $\Lambda \cap \Lambda^{\prime}$ is not empty and contains an interval of the form $\left(0, \lambda_{0}\right)$.

The main result here is:

Theorem 2.4. 1) $\Lambda \cap \Lambda^{\prime}$ is an open subset of $(0, \infty)$.

2) $\Lambda$ is closed.

3) $\Lambda$ contains the first connected component of $\Lambda^{\prime}$.

With this, to prove that $Q_{c_{\lambda}}$ is non-negative for all $\lambda$ reduces to the following:

Conjecture 2.5. For all positive $\lambda, \operatorname{ker} Q_{c_{\lambda}}=\left\langle\frac{\partial c_{\lambda}}{\partial x_{i}}, i=1,2,3\right\rangle$. 


\section{Convergence in the configuration space and Hessians.}

3.1. General Observations. The proof of the Theorem 2.4 relies on some general observations about quadratic forms from $[\mathbf{A D}]$. First, in order to describe the fact that the kernel of the Hessians changes as the solutions $c_{\lambda}$ move in the configuration space $\mathcal{C}$, adopt the following:

Definition. Let $(H,\langle\rangle$,$) be a Hilbert space. Let V_{\lambda_{0}}$ be a closed subspace of $H$ and $V_{\lambda}$ be a one-parameter family of closed subspaces of $H . V_{\lambda}$ contains $V_{\lambda_{0}}$ at the limit as $\lambda \rightarrow \lambda_{0}$ if for any $\varepsilon>0$ there exists $\delta>0$ such that $\left|\lambda-\lambda_{0}\right|<\delta$ implies that for any $u$ in $V_{\lambda}^{\perp}$ of norm 1 there exists $v$ in $V_{\lambda_{0}}^{\perp}$ of norm 1 such that $\|v-u\|<\varepsilon$.

Now, slightly abusing notation, let $Q_{\lambda_{0}}$ be a quadratic form and $Q_{\lambda}$ be a one-parameter family of quadratic forms on $H$. The following describes the steps for the proof of Theorem 2.4 in this general setting:

Proposition 3.1. Let $Q_{\lambda_{0}}$ be a quadratic form and $Q_{\lambda}$ be a one-parameter family of quadratic forms defined on a Hilbert space $H$ and assume that:

1) $Q_{\lambda_{0}}$ is uniformly continuous on the unit sphere of $H$.

2) $\alpha:=\inf \left\{Q_{\lambda_{0}}(v): v \perp \operatorname{ker} Q_{\lambda_{0}},\|v\|=1\right\} \supsetneqq 0$.

3) $\sup _{\|v\|=1}\left|Q_{\lambda_{0}}(v)-Q_{\lambda}(v)\right| \rightarrow 0$ as $\lambda \rightarrow \lambda_{0}$.

4) There are subspaces $N_{\lambda}$ of $\operatorname{ker} Q_{\lambda}$ such that $N_{\lambda}$ contains $\operatorname{ker} Q_{\lambda_{0}}$ at the limit as $\lambda \rightarrow \lambda_{0}$.

Then there exists $\varepsilon>0$ such that whenever $\left|\lambda-\lambda_{0}\right|<\varepsilon$ then

1) $\inf \left\{Q_{\lambda}(v): v \perp N_{\lambda},\|v\|=1\right\}>\frac{\alpha}{3} \supsetneqq 0$,

2) $N_{\lambda}=\operatorname{ker} Q_{\lambda}$.

Proof. If $v$ is in $N_{\lambda}^{\perp}$ and of norm 1 then, for $\lambda$ sufficiently close to $\lambda_{0}$, there is $v^{\prime}$ of norm 1 in $\operatorname{ker} Q_{c_{\lambda_{0}}}^{\perp}$ close to $v$. Therefore $Q_{c_{\lambda}}(v) \approx Q_{c_{\lambda_{0}}}(v) \approx Q_{c_{\lambda_{0}}}\left(v^{\prime}\right)>$ $\alpha / 3$.

3.2. Reduction to a single Hilbert space. Before Proposition 3.1 can take over, one has to establish that as $\lambda \rightarrow \lambda_{0}$ the spaces $T_{c_{\lambda}} \mathcal{C}$ are isomorphic and therefore all Hessians $Q_{c_{\lambda}}$ are defined on the same space.

Lemma 3.2. For any $c$ and $c^{\prime}$ in $\mathcal{C}$ with $c-c^{\prime}$ in $T_{c} \mathcal{C}$ the following holds:

$$
\left|\|v\|_{c^{\prime}}-\|v\|_{c}\right| \leq M_{c}\|v\|_{c}\left\|c-c^{\prime}\right\|_{c} .
$$

In particular, for $\left\|c-c^{\prime}\right\|_{c}$ sufficiently small the identity on $C_{0}^{\infty}$ induces an isomorphism between $T_{c} \mathcal{C}$ and $T_{c^{\prime}} \mathcal{C}$.

Proof. The proof for the norm $\|\cdot\|_{c}$ as defined here, is the same as the proof of Proposition B6.2 of [T1].

That the conditions of this Lemma are satisfied for spherically symmetric solutions is proved in the following: 
Proposition 3.3. $\left\|c_{\lambda}-c_{\lambda_{0}}\right\|_{c_{\lambda_{0}}} \rightarrow 0$, as $\lambda \rightarrow \lambda_{0}>0$.

Proof. It is a matter of straightforward calculation to show that this follows by the fact that the following norms over $[0, \infty)$ go to 0 as $\lambda \rightarrow \lambda_{0}$ :

$$
\begin{gathered}
\left\|\frac{1}{r}\left(H_{\lambda}-H_{\lambda_{0}}\right)\right\|_{2}, \quad\left\|H_{\lambda}^{\prime}-H_{\lambda_{0}}^{\prime}\right\|_{2}, \quad\left\|\left(H_{\lambda}-H_{\lambda_{0}}\right)\right\|_{2} . \\
\left\|K_{\lambda}-K_{\lambda_{0}}\right\|_{2}, \quad\left\|\frac{1}{r}\left(K_{\lambda}-K_{\lambda_{0}}\right)\right\|_{2}, \quad\left\|K_{\lambda}^{\prime}-K_{\lambda_{0}}^{\prime}\right\|_{2} .
\end{gathered}
$$

For these estimates work as follows:

Step 1. Estimates for $H_{\lambda}$ and $K_{\lambda}$. First obtain uniform in $\lambda$ pointwise bounds on the fields on $\left[0, r_{0}\right)$, for $r_{0}$ sufficiently small, see (23) and (24) in Section 4.

Then obtain uniform in $\lambda$ pointwise exponential decay estimates on the fields on $\left[r_{1}, \infty\right)$ for $r_{1}$ sufficiently large, see Proposition 4.2 in Section 4.

For the intervals of the form $\left[r_{0}, r_{1}\right]$, Proposition 7.1 of $[\mathbf{A D}]$ shows that $\left\|F_{A_{\lambda}}\right\|_{2}+\left\|d_{A_{\lambda} \Phi_{\lambda}}\right\|_{2}$ is an non-decreasing function of $\lambda$, therefore bounded on bounded intervals. As an elementary case of Uhlenbeck's compactness, this suffices for uniform convergence of the fields on bounded domains, see Proposition 7.4 of $[\mathrm{AD}]$.

Step 2. Estimates for $H_{\lambda}^{\prime \prime}$ and $K_{\lambda}^{\prime \prime}$. These follow from the estimates on $H_{\lambda}$ and $K_{\lambda}$ after using equations (YMH1) and (YMH2) that do not involve first derivatives, see Proposition 4.1 below.

Step 3. Estimates for $H_{\lambda}^{\prime}$ and $K_{\lambda}^{\prime}$. For these, obtain uniform in $\lambda$ pointwise decay estimates on the first derivatives on $\left[r_{1}, \infty\right)$, c.f. Propositions 8.2, 9.4 and 9.5 of $[\mathbf{A D}]$. On $\left[0, r_{1}\right)$ use Step 2 , the Poincaré inequality and the fact that $H_{\lambda}^{\prime}(0)=K_{\lambda}^{\prime}(0)=0$.

The details follow from the arguments in $[\mathbf{A D}]$, after observing that the pointwise estimates there on the fields $H_{\lambda}, K_{\lambda}$ and their first derivatives are valid for $\lambda$ in any specified bounded interval; see Section 4 below.

Remark. $\left\|H_{\lambda}-H_{\lambda_{0}}\right\|_{2} \rightarrow 0$ does not hold for $\lambda_{0}=0$ over $[0, \infty)$ but only over compact intervals. This reflects the fact that $H_{0}$ decays in power law whereas $H_{\lambda}$ decays exponentially for all $\lambda>0$, see Proposition 4.2.

The remaining subsections of this section show that the conditions of Proposition 3.1 hold for the Hessians $Q_{c_{\lambda}}$.

\subsection{Uniform continuity of $Q_{\lambda, c}$ on the unit sphere.}

Lemma 3.4. For any $\lambda$, and for any $c=(A, \Phi)$ in $\mathcal{C}$ with $\Phi$ bounded the Hessian $Q_{\lambda, c}:\left(T_{c} \mathcal{C},\|\cdot\|_{c}\right) \rightarrow \mathbf{R}$ is uniformly continuous on the unit sphere. 
Proof. It suffices to show that $\hat{Q}_{\lambda, c}(v, w) \leq K\|v\|_{c} \cdot\|w\|_{c}$ for some constant $K$ and all $v$ and $w$. To see that this holds separately for each term of $\hat{Q}_{\lambda, c}$, use the fact that $\Phi$ is bounded and the inequalities

$$
\begin{gathered}
\left|\left\langle F_{A},[a, a]\right\rangle\right| \leq\left\|F_{A}\right\|_{2}\|[a, a]\|_{2} \leq C\left\|F_{A}\right\|_{2}\|(a, \phi)\|_{c}^{2}, \\
\left|\left\langle d_{A} \Phi,[a, \phi]\right\rangle\right| \leq\left\|d_{A} \Phi\right\|_{2}\|[a, \phi]\|_{2} \leq C\left\|d_{A} \Phi\right\|_{2}\|(a, \phi)\|_{c}^{2},
\end{gathered}
$$

c.f. $[\mathbf{T} 1]$.

Remark. It is standard to show using maximum principle that for any critical point $c=(A, \Phi)$ of $E_{\lambda}$, and in particular for the spherically symmetric solutions $c_{\lambda}$, the Higgs field $\Phi$ satisfies $|\Phi|(x)<1$.

\subsection{When $Q_{\lambda, c}$ is non-negative.}

Theorem 3.5. For any $c$ in $\mathcal{C}$ the following fold:

1) If $\Phi$ is bounded then $Q_{\lambda, c}:\left(T_{c} \mathcal{C},\|.\|_{c}\right) \rightarrow \mathbf{R}$ is continuously differentiable.

2) There is $\varepsilon>0$ such that $Q_{\lambda, c}-\varepsilon\|.\|_{c}^{2}$ is weakly lower semi-continuous in $\left(T_{c} \mathcal{C},\|\cdot\|_{c}\right)$.

Proof. 1) Since $\hat{Q}_{\lambda, c}$ is continuous, this follows from the fact that $2 \hat{Q}_{\lambda, c}(v,$. is the differential of $Q_{\lambda, c}$ at $v$.

2) First use Lemma 2.2 to rewrite the Hessian as

$$
\begin{aligned}
Q_{\lambda, c}(a, \phi)= & \left\|\nabla_{A} a\right\|_{2}^{2}+\left\|d_{A} \phi\right\|_{2}^{2}+\|[a, \Phi]\|_{2}^{2}+\|[\Phi, \phi]\|_{2}^{2} \\
& +2\left\langle F_{A},[a, a]\right\rangle+4\left\langle d_{A} \Phi,[a, \phi]\right\rangle \\
& +\frac{\lambda}{2} \int_{\mathbf{R}^{3}}\left(|\Phi|^{2}-1\right)|\phi|^{2} d^{3} x+\lambda \int_{\mathbf{R}^{3}}\langle\Phi, \phi\rangle^{2} d^{3} x .
\end{aligned}
$$

Then for $\varepsilon \leq \min (\lambda, 1)$ we have:

$$
\begin{aligned}
& Q_{\lambda, c}(a, \phi)-\varepsilon\|(a, \phi)\|_{c}^{2} \\
& =(1-\varepsilon)\left\|\nabla_{A} a\right\|_{2}^{2}+(1-\varepsilon)\left\|d_{A} \phi\right\|_{2}^{2} \\
& \quad+(1-\varepsilon)\|[a, \Phi]\|_{2}^{2}+(1-\varepsilon)\|[\phi, \Phi]\|_{2}^{2}+(\lambda-\varepsilon) \int_{\mathbf{R}^{3}}\langle\Phi, \phi\rangle^{2} d^{3} x \\
& \quad+2\left\langle F_{A},[a, a]\right\rangle+4\left\langle d_{A} \Phi,[a, \phi]\right\rangle+\frac{\lambda}{2} \int_{\mathbf{R}^{3}}\left(|\Phi|^{2}-1\right)|\phi|^{2} d^{3} x \\
& \quad+\varepsilon\|[\phi, \Phi]\|_{2}^{2}+\varepsilon \int_{\mathbf{R}^{3}}\langle\Phi, \phi\rangle^{2} d^{3} x-\varepsilon\|\phi\|_{2}^{2} .
\end{aligned}
$$

Each term of the first two lines is weakly lower semi-continuous with respect to the $\|\cdot\|_{c}$-norm: For the first term, for example, note that if $\left(a_{n}, \phi_{n}\right)$ converges weakly to $(a, \phi)$ in $T_{c} \mathcal{C}$ then

$$
\left\|\nabla_{A} a\right\|_{2} \leq\|(a, \phi)\|_{c} \leq \liminf _{n \rightarrow \infty}\left\|\left(a_{n}, \phi_{n}\right)\right\|_{c}
$$


since $\|\cdot\|_{c}$ is a norm and hence weakly lower semicontinuous.

The weak continuity of the terms in the third line follows as in Section VI of [T2] from the fact that $F_{A}, d_{A} \Phi$ and $\left.|| \Phi\right|^{2}-1 \mid$ belong to $L^{2}$ (and therefore Property $(*)$ of $[\mathbf{T} 2]$ is satisfied).

The terms in the fourth line are also weakly continuous since they regroup as

$$
\varepsilon \int_{\mathbf{R}^{3}}\left(|\Phi|^{2}-1\right)|\phi|^{2} d^{3} x
$$

The following is the main application of the weak lower semi-continuity offered by Theorem 3.5.

Proposition 3.6. For any $\lambda$, if $Q_{\lambda, c}(v) \geq 0$ for all $v$ in $T_{c} \mathcal{C}$ then

$$
\inf \left\{Q_{\lambda, c}(v): v \perp_{c} \operatorname{ker} Q_{\lambda, c},\|v\|_{c}=1\right\} \supsetneqq 0 .
$$

Proof. Let $v_{n}$ be a sequence in $\operatorname{ker} Q_{\lambda, c}^{\perp}$ with $\left\|v_{n}\right\|_{c}=1$ and $Q_{\lambda, c}\left(v_{n}\right) \rightarrow 0$. By considering a subsequence, assume that $v_{n}$ is weakly convergent and let $v \in \operatorname{ker} Q_{\lambda, c}^{\perp}$ be its weak limit. Since $Q_{\lambda, c}$ is weakly lower semicontinuous (by Theorem 3.5) and non-negative, obtain that $Q_{\lambda, c}(v)=0$. Moreover, since there is an $\varepsilon>0$ such that $Q_{\lambda, c}(\cdot)-\varepsilon\|\cdot\|_{c}^{2}$ is weakly lower semicontinuous, it follows that

$$
-\varepsilon\|v\|_{c}^{2}=Q_{\lambda, c}(v)-\varepsilon\|v\|_{c}^{2} \leq \liminf _{n}\left(Q_{\lambda, c}\left(v_{n}\right)-\varepsilon\left\|v_{n}\right\|_{c}^{2}\right)=-\varepsilon
$$

which implies that $\|v\|_{c} \geq 1$ and thus $\|v\|_{c}=1$ (therefore $\left(v_{n}\right)$ converges to $v$ in the Hilbert space norm $\|\cdot\|_{c}$ ). Hence

$$
Q_{\lambda, c}(v)=\min \left\{Q_{\lambda, c}(u): u \perp_{c} \operatorname{ker} Q_{\lambda, c},\|u\|_{c}=1\right\} .
$$

Then by the differentiability and the non-negativity of $Q_{\lambda, c}$ it is easy to see using a Lagrange multiplier that for each $w \in \operatorname{ker} Q_{\lambda, c}^{\perp}$

$$
\hat{Q}_{\lambda, c}(v, w)=Q_{\lambda, c}(v)\langle v, w\rangle_{c}
$$

which gives that $v$ is in $\operatorname{ker} Q_{\lambda, c}$, a contradiction to the fact that $v \in \operatorname{ker} Q_{\lambda, c}^{\perp}$ of norm 1.

\subsection{Uniform convergence on the sphere.}

Lemma 3.7. For $c_{i}=\left(A_{i}, \Phi_{i}\right)$ and $c=(A, \Phi)$ in $\mathcal{C}$ assume that $\left\|c_{i}-c\right\|_{c} \rightarrow$ 0 . If $\lambda_{i}$ converge to $\lambda,\left|\Phi_{i}-\Phi\right|$ tends to zero uniformly on $\mathbf{R}^{3}$ and $|\Phi|$ is bounded on $\mathbf{R}^{3}$, then

$$
\sup _{\|v\|_{c}=1}\left|Q_{\lambda_{i}, c_{i}}(v)-Q_{\lambda, c}(v)\right| \rightarrow 0 .
$$


Proof. For the part of the Hessian with no $\lambda$ coefficient, see statement (5) of Proposition A.4.3. of [T3]. For the remaining terms note that

$$
\begin{aligned}
& \mid \lambda_{i}\left(\frac{1}{2} \int\left(\left|\Phi_{i}\right|^{2}-1\right)|\phi|^{2} d^{3} x+\int\left\langle\Phi_{i}, \phi\right\rangle^{2} d^{3} x\right) \\
& \quad-\lambda\left(\frac{1}{2} \int\left(|\Phi|^{2}-1\right)|\phi|^{2} d^{3} x+\int\langle\Phi, \phi\rangle^{2} d^{3} x\right) \mid \\
& \leq\left|\lambda_{i}-\lambda\right| \frac{1}{2} \int\left(|\Phi|^{2}-1\right)|\phi|^{2} d^{3} x+\left.\left|\lambda_{i}\right| \frac{1}{2} \int|| \Phi_{i}\right|^{2}-\left.|\Phi|^{2}|| \phi\right|^{2} d^{3} x \\
& \quad+\left|\lambda_{i}-\lambda\right| \int\langle\Phi, \phi\rangle^{2} d^{3} x+\left|\lambda_{i}\right| \int\left|\left\langle\Phi_{i}, \phi\right\rangle^{2}-\langle\Phi, \phi\rangle^{2}\right| d^{3} x .
\end{aligned}
$$

The first and third term in the last expression obviously tend to zero. The second term tends to zero since $\Phi_{i}$ tends to $\Phi$ uniformly on $\mathbf{R}^{3}$ and $\|\phi\|_{2} \leq 1$. The fourth term tends to zero since

$$
\int\left|\left\langle\Phi_{i}, \phi\right\rangle^{2}-\langle\Phi, \phi\rangle^{2}\right| d^{3} x \leq \int\left|\Phi_{i}-\Phi\right||\phi|^{2}\left|\Phi_{i}+\Phi\right| d^{3} x
$$

and $\left|\Phi_{i}+\Phi\right|$ is bounded on $\mathbf{R}^{3}$.

Lemma 3.8. For $\lambda_{0} \geq 0, \Phi_{\lambda}$ tends to $\Phi_{\lambda_{0}}$ uniformly on $\mathbf{R}^{3}$ as $\lambda \rightarrow \lambda_{0}$.

Proof. First note that in terms of $H_{\lambda}$ it is enough to show that as $\lambda \rightarrow \lambda_{0}$

$$
\left\|\frac{H_{\lambda}}{r}-\frac{H_{\lambda_{0}}}{r}\right\|_{\infty} \rightarrow 0
$$

For this use Corollary 4.3 for large $r$, estimate (24) as it appears in the proof of Proposition 4.1 for small $r$, and the uniform convergence on compact intervals (as in Step 1, Proposition 3.3) in between.

3.6. $\lambda$-subspaces containing the kernel of $Q_{c_{\lambda_{0}}}$ at the limit. For $\lambda \geq 0$ consider the subspace of $T_{c_{\lambda}} \mathcal{C}$ spanned by the translation modes, i.e., the partial derivatives of $c_{\lambda}$ :

$$
N_{\lambda}=\left\langle\frac{\partial c_{\lambda}}{\partial x_{i}}, i=1,2,3\right\rangle .
$$

As a result of a straight-forward calculation

$$
\begin{aligned}
& \left.\frac{d^{2}}{d s d t}\right|_{t=0, s=0} E_{\lambda}\left(c\left(x+t e_{i}\right)+s v\left(x+t e_{i}\right)\right) \\
& =\hat{Q}_{\lambda, c}\left(\frac{\partial c}{\partial x_{i}}, v\right)+\nabla\left(E_{\lambda}\right)_{c}\left(\frac{\partial v}{\partial x_{i}}\right)
\end{aligned}
$$

for any $v$ in $T_{c} \mathcal{C}$. Since $E_{\lambda}$ is translation invariant

$$
\frac{d}{d t} E_{\lambda}\left(c\left(x+t e_{i}\right)+s v\left(x+t e_{i}\right)\right)=0 .
$$


In addition the first variation of $E_{\lambda}$ vanishes at $c_{\lambda}$, therefore

$$
Q_{c_{\lambda}}\left(\frac{\partial c_{\lambda}}{\partial x_{i}}, v\right)=0
$$

which shows that $N_{\lambda}$ is a subspace $\operatorname{ker} Q_{c_{\lambda}}$.

Proposition 3.9. $N_{\lambda}$ contain $S_{\lambda_{0}}$ at the limit in $\left(T_{c_{\lambda_{0}}} \mathcal{C},\|\cdot\|_{c_{\lambda_{0}}}\right)$ as $\lambda \rightarrow$ $\lambda_{0} \neq 0$.

Proof. As a matter of a straightforward calculation using that $\left|\Phi_{\lambda_{0}}\right|(x)<1$, and that $\left|A_{\lambda_{0}}\right|$ is uniformly bounded by $[\mathbf{A D}]$,

$$
\left\|\frac{\partial c_{\lambda}}{\partial x_{a}}-\frac{\partial c_{\lambda_{0}}}{\partial x_{a}}\right\|_{c_{\lambda_{0}}}^{2} \rightarrow 0
$$

if, in addition to the estimates in the proof of 3.3 , the following norms over $[0, \infty)$ tend to 0 as $\lambda \rightarrow \lambda_{0}$ :

$$
\begin{aligned}
& \left\|\frac{1}{r^{2}}\left(H_{\lambda}-H_{\lambda_{0}}\right)\right\|_{2}, \quad\left\|H_{\lambda}^{\prime \prime}-H_{\lambda_{0}}^{\prime \prime}\right\|_{2}, \\
& \qquad\left\|\frac{1}{r}\left(H_{\lambda}^{\prime}-H_{\lambda_{0}}^{\prime}\right)\right\|_{2}, \quad\left\|K_{\lambda}^{\prime \prime}-K_{\lambda_{0}}^{\prime \prime}\right\|_{2} .
\end{aligned}
$$

Observe that this involves estimates on the fields, their first and their second derivatives. These follow again as in $[\mathrm{AD}]$ using the fact that the pointwise estimates on the fields and their derivatives hold for $\lambda$ on any specified bounded interval, see Section 4.

Proof or Theorem 2.4. Fix $\lambda_{0}$ in $\Lambda \cap \Lambda_{0}$. Then subsections 3.2 to 3.6 show that $Q_{c_{\lambda_{0}}}$ satisfies the conditions 1) to 4) respectively of Proposition 3.1, and that for $\lambda$ close to $\lambda_{0}$ all the Hessians $Q_{c_{\lambda}}$ are defined on the same space as $Q_{c_{\lambda_{0}}}$. Then Proposition 3.1 applies to give part 1) of Theorem 2.4.

The second part of Theorem 2.4 follows immediately from Lemma 3.7, which shows that the complement of $\Lambda$ is open.

Now for the third part of Theorem 2.4 argue as follows: by Thorem 2.3 , both the first connected component $\Lambda_{0}$ of $\Lambda$ and the first connected component $\Lambda_{0}^{\prime}$ of $\Lambda^{\prime}$ are intervals starting from 0 . If $\Lambda_{0}^{\prime}$ is not contained in $\Lambda$ then $\Lambda_{0}$ is a proper subset of $\Lambda_{0}^{\prime}$. Let $\lambda_{0}$ be the supremum of $\Lambda_{0}$, which by the second part of the theorem belongs to $\Lambda_{0}$, i.e., $\lambda_{0}$ is in the intersection of $\Lambda$ and $\Lambda^{\prime}$. This contradicts the first part of the theorem. The proof of theorem 2.4 is now complete.

\section{Estimates.}

This section will substantiate the claim that the estimates of $[\mathbf{A D}]$ for $\lambda$ in a neighborhood of 0 can be extended to estimates on any bounded $\lambda$-interval. 
Proposition 4.1 is typical of the $L^{2}$-norm estimates required. Proposition 4.2 is typical of the uniform in $\lambda$ and pointwise in $r$ estimates required.

Proposition 4.1. $\left\|H_{\lambda}^{\prime \prime}-H_{\lambda_{0}}^{\prime \prime}\right\|_{L^{2}[0, \infty)} \rightarrow 0$, as $\lambda \rightarrow \lambda_{0} \neq 0$.

Proof. For this, first note that it follows as in Section 10 of [AD] that there is a constant $C$ such that for all $\lambda<\lambda_{0}+1$

$$
\left|K_{\lambda}(r)\right| \leq C
$$

for all $r$ and

$$
\left|\frac{H_{\lambda}(r)}{r^{2}}\right| \leq C
$$

for $r$ in $[0,1]$. Then

$$
\begin{aligned}
& \left\|H_{\lambda}^{\prime \prime}-H_{\lambda_{0}}^{\prime \prime}\right\|_{L^{2}[0, \infty)} \\
& =\left\|\frac{2 K_{\lambda}^{2} H_{\lambda}}{r^{2}}-\frac{2 K_{\lambda_{0}}^{2} H_{\lambda_{0}}}{r^{2}}-4 \lambda H_{\lambda}\left(1-\frac{H_{\lambda}^{2}}{r^{2}}\right)-4 \lambda_{0} H_{\lambda_{0}}\left(1-\frac{H_{\lambda_{0}}^{2}}{r^{2}}\right)\right\|_{L^{2}[0, \infty)} \\
& \leq\left\|\frac{2 K_{\lambda}^{2} H_{\lambda}}{r^{2}}-\frac{2 K_{\lambda_{0}}^{2} H_{\lambda_{0}}}{r^{2}}\right\|_{L^{2}[0, \infty)} \\
& +\left\|4 \lambda H_{\lambda}\left(1-\frac{H_{\lambda}^{2}}{r^{2}}\right)-4 \lambda_{0} H_{\lambda_{0}}\left(1-\frac{H_{\lambda_{0}}^{2}}{r^{2}}\right)\right\|_{L^{2}[0, \infty)} .
\end{aligned}
$$

For the first term in this sum find appropriately small $r_{0}$ and large $r_{1}$ such that:

$$
\begin{aligned}
\| & \frac{2 K_{\lambda}^{2} H_{\lambda}}{r^{2}}-\frac{2 K_{\lambda_{0}}^{2} H_{\lambda_{0}}}{r^{2}}\left\|_{L^{2}[0, \infty)}+\right\| \frac{K_{\lambda_{0}}^{2} H_{\lambda_{0}}}{r^{2}} \|_{L^{2}\left[0, r_{0}\right]} \\
\leq & \left\|\frac{K_{\lambda}^{2} H_{\lambda}}{r^{2}}\right\|_{L^{2}\left[0, r_{0}\right]}+\left\|\frac{\left(K_{\lambda}^{2}-K_{\lambda_{0}}^{2}\right) H_{\lambda_{0}}}{r^{2}}\right\|_{L^{2}\left[r_{0}, r_{1}\right]} \\
& +\left\|\frac{K_{\lambda}^{2}\left(H_{\lambda}-H_{\lambda_{0}}\right)}{r^{2}}\right\|_{L^{2}\left[r_{0}, r_{1}\right]}+\left\|\frac{e^{-r / 2}}{r}\right\|_{L_{2}\left[r_{1}, \infty\right)} \\
& +2 \| C^{3} M \varepsilon+C^{3} \varepsilon \\
& +\frac{C^{2}}{r_{0}^{2}}\left\|H_{\lambda}-H_{\lambda_{0}}\right\|_{L^{2}\left[r_{0}, r_{1}\right]}+\frac{r_{1}}{r_{0}^{2}}\left\|K_{\lambda}^{2}-K_{\lambda_{0}}^{2}\right\|_{L^{2}\left[r_{0}, r_{1}\right]} \\
& +2 \varepsilon .
\end{aligned}
$$


As already remarked in Step 1. of Proposition 3.3 above, $E_{0}$ is an increasing function of $\lambda$, and therefore bounded on a bounded interval, and that this is enough to give uniform convergence of $H_{\lambda}$ to $H_{0}$ and of $K_{\lambda}$ to $K_{0}$ on bounded domains. Therefore the last expression becomes smaller than $5 \varepsilon$ if $\left|\lambda-\lambda_{0}\right|$ is small enough, by the uniform convergence of $K_{\lambda}$ to $K_{\lambda_{0}}$ and $H_{\lambda_{0}}$ to $H_{0}$ on the interval $\left[r_{0}, r_{1}\right]$.

For the second term and for a choice of $0<r_{0}<r_{1}$,

$$
\begin{aligned}
& \| 4 H_{\lambda}\left(1-\frac{H_{\lambda}^{2}}{r^{2}}\right) \\
& -4 \lambda_{0} H_{\lambda_{0}}\left(1-\frac{H_{\lambda_{0}}^{2}}{r^{2}}\right)\left\|_{L^{2}[0, \infty)}+\right\| 4 \lambda_{0} H_{\lambda_{0}}\left(1-\frac{H_{\lambda_{0}}^{2}}{r^{2}}\right) \|_{L^{2}\left[0, r_{0}\right]} \\
& \leq\left\|4 \lambda H_{\lambda}\left(1-\frac{H_{\lambda}^{2}}{r^{2}}\right)\right\|_{L^{2}\left[0, r_{0}\right]}\|\|_{L^{2}\left[r_{0}, r_{1}\right]} \\
& +4\left\|\left(\lambda-\lambda_{0}\right) H_{\lambda}\left(1-\frac{H_{\lambda}^{2}}{r^{2}}\right)\right\| \|_{L^{2}\left[r_{0}, r_{1}\right]} \\
& +4 \| \lambda_{0}\left(H_{\lambda}-H_{\lambda_{0}}\right)\left(1-\frac{H_{\lambda_{0}}}{r^{2}}\left(H_{\lambda_{0}}^{2}-H_{\lambda}^{2}\right) \|_{L^{2}\left[r_{0}, r_{1}\right]}\right. \\
& +4\left\|\lambda_{0}\left(H_{\lambda}-H_{\lambda_{0}}\right)\left(1-\frac{H_{\lambda}^{2}}{r^{2}}\right)\right\|_{L^{2}\left[r_{1}, \infty\right)} \\
& +4\left\|\lambda_{0} \frac{H_{\lambda_{0}}}{r}\left(\left(1-\frac{H_{\lambda}}{r}\right)\left(1-\frac{H_{\lambda_{0}}}{r}\right)\right)\left(H_{\lambda_{0}}+H_{\lambda}\right)\right\|_{L^{2}\left[r_{1}, \infty\right)}
\end{aligned}
$$

Obviously, $r_{0}$ can be chosen small enough to make the first two terms arbitrarily small. Then $r_{1}$ can be chosen large enough to make the last two terms arbitrarily small (for the last term use triangle inequality of the norm and Proposition 4.2; for the anti-penultimate term use Corollary 4.3 and Proposition 4.2). The rest of the terms tend to zero as $\lambda \rightarrow \lambda_{0}$ by uniform convergence on compact intervals.

Proposition 4.2. For all $\lambda_{0} \geq 0$ there exist $\alpha>0, r_{0}>0$ such that for all $\lambda \in\left[\lambda_{0}-1, \lambda_{0}+1\right] \cap[0, \infty)$ and for all $r \geq r_{0}$

$$
\left|1-\frac{H_{\lambda}(r)}{r}\right| \leq \alpha e^{-\min (2 \sqrt{\lambda}, 1) r} .
$$


Proof. Let $u_{\lambda}(r)=1-\frac{H_{\lambda}(r)}{r}$. Differentiate twice to obtain

$$
u_{\lambda}^{\prime \prime}+\frac{2}{r} u_{\lambda}^{\prime}=-\frac{H_{\lambda}^{\prime \prime}}{r} .
$$

Replacing $H_{\lambda}^{\prime \prime}$ from (YMH-2) yields

$$
u_{\lambda}^{\prime \prime}+\frac{2}{r} u_{\lambda}^{\prime}-\frac{4 \lambda H_{\lambda}}{r}\left(1+\frac{H_{\lambda}}{r}\right) u_{\lambda}=-\frac{2 K_{\lambda}^{2} H_{\lambda}}{r^{3}} .
$$

Now let

$$
s(r)=\alpha e^{-\min (2 \sqrt{\lambda}, 1) r}
$$

to be the test function. The aim is to show that there exist $\alpha \geq 1$ and $r_{0}>0$ such that

$$
\left|u_{\lambda}(r)\right| \leq s(r)
$$

for all $r \geq r_{0}$ and for all $\lambda$ in an appropriate range. Since

$$
\left|1-\frac{H_{\lambda}(r)}{r}\right| \leq \sqrt{\frac{C}{r}}
$$

and

$$
\left|K_{\lambda}(r)\right| \leq \alpha e^{-r / 2},
$$

(see $[\mathbf{A D}])$, for $\lambda \in\left[\lambda_{0}-1, \lambda_{0}+1\right] \cap[0, \infty)$

$$
\begin{aligned}
&\left(s \pm u_{\lambda}\right)^{\prime \prime}+\frac{2}{r}(s \pm u)^{\prime}-\frac{4 \lambda H_{\lambda}}{r}\left(1+\frac{H_{\lambda}}{r}\right)\left(s \pm u_{\lambda}\right) \\
&=\mp \frac{2 K_{\lambda}^{2} H_{\lambda}}{r^{3}}+\alpha \min (4 \lambda, 1) e^{-\min (2 \sqrt{\lambda}, 1) r}-\frac{2}{r} \alpha \min (2 \sqrt{\lambda}, 1) e^{-\min (2 \sqrt{\lambda}, 1) r} \\
& \quad-\frac{4 \lambda H_{\lambda}}{r}\left(1+\frac{H_{\lambda}}{r}\right) \alpha e^{-\min (2 \sqrt{\lambda}, 1) r} \\
& \leq \frac{2 e^{-r}}{r^{2}}+\alpha e^{-\min (2 \sqrt{\lambda}, 1) r} \\
&-4 \lambda\left(1-\sqrt{\frac{C}{r}}\right)\left(1+1-\sqrt{\frac{C}{r}}\right) \alpha e^{-\min (2 \sqrt{\lambda}, 1) r} \\
& \leq \frac{2 e^{-r}}{r^{2}}+\alpha e^{-\min (2 \sqrt{\lambda}, 1) r} \\
&-8 \lambda \alpha e^{-\min (2 \sqrt{\lambda}, 1) r}+12\left(\lambda_{0}+1\right) \sqrt{\frac{C}{r}} \alpha e^{-\min (2 \sqrt{\lambda}, 1) r} .
\end{aligned}
$$

Note that the term $-8 \lambda \alpha e^{-\min (2 \sqrt{\lambda}, 1) r}$ is negative and it eventually makes the above expression negative as well since $\left|\lambda-\lambda_{0}\right| \leq 1$, i.e., there exists 
$r_{0}>0$ such that the last expression is negative for all $r \geq r_{0}$ and for all $\lambda \in\left[\lambda_{0}-1, \lambda_{0}+1\right] \cap[0, \infty)$. Since

$$
u_{\lambda}\left(r_{0}\right) \leq 1+\sqrt{\frac{C}{r_{0}}}
$$

for all $\lambda$ in the range $\left[\lambda_{0}-1, \lambda_{0}+1\right] \cap[0, \infty)$, choose $\alpha \geq 1$ such that

$$
s_{\lambda}\left(r_{0}\right) \pm u_{\lambda}\left(r_{0}\right)>0
$$

for all such $\lambda$ 's.

Corollary 4.3. For every $\lambda_{0}>0$ there exists $M>0$ such that for $\lambda$ in $\left[0, \lambda_{0}\right]$ the following holds for $r \geq 0$

$$
\left|H_{\lambda}(r)-H_{\lambda_{0}}(r)\right| \leq M
$$

\section{References}

[AD] G. Androulakis and S. Dostoglou, On the stability of monopole solutions, Nonlinearity, 11(3) (1998), 377-408.

[D] S. Dostoglou, Remarks on the Existence of Solutions of the Yang-MillsHiggs Equations with Arbitrary Higgs Potential, Preprint available at http://www.math.missouri.edu/people/sdostoglou.html

[H] P. Higgs, Spontaneous symmetry breakdown without massless boson, Phys. Rev., 145 (1966), 1156-1163.

[tH] G. 't Hooft, Magnetic monopoles in unified gauge theories, Nucl. Phys. B, 79 (1976), 276.

[JT] A. Jaffe and C.H. Taubes, Vortices and Monopoles, Birkhauser, 1980.

[L] H.B. Lawson, The theory of gauge fields in four dimensions, CBMS Regional Conference Series in Mathematics, 58; American Mathematical Society, Providence, R.I., 1985.

[M] D. Maison, Uniqueness of the Prasad-Sommerfield monopole solution, Nucl. Phys. B, 182 (1981), 144-150.

$[\mathrm{Pa}]$ R.S. Palais, The principle of symmetric criticality, Comm. Math. Phys., 69(1) (1979), 19-30.

[P] A.M. Polyakov, JETP Letters, 20, 1974, 194.

[T1] C.H. Taubes, Monopoles and maps from $S^{2}$ to $S^{2}$; the topology of the configuration space, Commun. Math. Phys., 95 (1984), 345-391.

[T2] _ Stability in Yang-Mills Theories, Commun. Math. Phys., 91 (1983), 235263.

[T3] _ Existence of a non-minimal solutions to the SU(2) Yang-Mills-Higgs equations, Part I, Comm. Math. Phys., 86 (1982), 257-298. 
[WB] A. Wirzba and H. Bang, The mode spectrum and stability analysis of skyrmions on the 3-sphere, Nuclear Phys. A, 515(4) (1990), 571- 598.

Received June 17, 1998 and revised July 20, 1999.

University of Missouri-Columbia

Columbia, MO 65211

E-mail address: giorgis@math.missouri.edu

University of Missouri-Columbia

Columbia, MO 65211

E-mail address: stamatis@euler.math.missouri.edu 